ARTICLE OPEN

\title{
Giant nonlinear Hall effect in twisted bilayer $\mathrm{WTe}_{2}$
}

\author{
Zhihai $\mathrm{He}^{1,2}$ and Hongming Weng (1D ${ }^{1,2,3 凶}$
}

In a system with broken inversion symmetry, a second-order nonlinear Hall effect can survive even in the presence of time-reversal symmetry. In this work, we show that a giant nonlinear Hall effect can exist in twisted bilayer WTe ${ }_{2}$ system. The Berry curvature dipole of twisted bilayer $\mathrm{WTe}_{2}\left(\theta=29.4^{\circ}\right)$ can reach up to $\sim 1400 \AA$, which is much larger than that in previously reported nonlinear Hall systems. In twisted bilayer WTe $\mathrm{W}_{2}$ system, there exist abundant band anticrossings and band inversions around the Fermi level, which brings a complicated distribution of Berry curvature, and leads to the nonlinear Hall signals that exhibit dramatically oscillating behavior in this system. Its large amplitude and high tunability indicate that the twisted bilayer WTe $\mathrm{C}_{2}$ can be an excellent platform for studying the nonlinear Hall effect.

npj Quantum Materials (2021)6:101 ; https://doi.org/10.1038/s41535-021-00403-9

\section{INTRODUCTION}

Over the last few decades, the study of various Hall effects has become an important topic in condensed-matter physics. For Hall effect or anomalous Hall effect, it is well known that an external magnetic field or magnetic dopants are necessary to break the time-reversal symmetry ${ }^{1}$. However, a recent theoretical study predicted that a second-order nonlinear Hall effect can exist in materials with time-reversal symmetry but without inversion symmetry ${ }^{2}$. Subsequently, the experimental study on bilayer and multilayer $\mathrm{WTe}_{2}$ has validated this prediction in terms of the observed transverse nonlinear Hall-like current with a quadratic current-voltage characteristic ${ }^{3,4}$. The nonlinear Hall effect arises from the non-vanishing dipole moment of Berry curvature in momentum space, i.e. Berry curvature dipole ${ }^{2}$. The theoretical predictions of the nonlinear Hall effect in a material can be achieved by calculating the Berry curvature dipole from band structure. Multiple materials ${ }^{5-17}$ have been predicted or validated to possess strong nonlinear Hall effect, such as the Weyl semimetals ${ }^{5-8}$, the giant Rashba material bismuth tellurium iodine (BiTel) under pressure ${ }^{10}$, the monolayer $\mathrm{WTe}_{2}$ and $\mathrm{MoTe}_{2}$ with an external electric field ${ }^{11}$, and the strained twisted bilayer graphene $^{12}$ or $\mathrm{WSe}_{2}{ }^{13,14}$.

The experimental observations of the nonlinear Hall effect are mainly limited to the two-dimensional systems $s^{3,4,14-16}$. It is well known that the twist angle between adjacent layers can be used as a new degree of freedom to modulate the electronic structures of two-dimensional system, which has attracted great attention since the recent discovery of the unconventional superconductivity and correlated state in the twisted bilayer graphene ${ }^{18,19}$ and twisted bilayer of transition metal dichalcogenides ${ }^{20}$. Very recently, it was shown that the strained twisted bilayer graphene ${ }^{12}$ and $\mathrm{WSe}_{2}{ }^{13,14}$ present a large nonlinear Hall response. The experimental results demonstrate that there exists a significant nonlinear Hall effect in bilayer $\mathrm{WTe}_{2}{ }^{3}$, it is interesting to know how the twist regulates the nonlinear Hall effect in this system.

In this work, we study the nonlinear Hall effect in twisted bilayer $\mathrm{WTe}_{2}$ by using first-principles calculations combined with the semiclassical approach. Multiple twisted bilayer $\mathrm{WTe}_{2}$ structures are constructed, and the twist angles range from $12^{\circ}$ to $73^{\circ}$. It is found that the twisted bilayer $\mathrm{WTe}_{2}$ has a more complicated band structure compared to the prefect bilayer system. We choose the twisted bilayer $\mathrm{WTe}_{2}$ with twist angle $\theta=29.4^{\circ}$ as a typical system, and show that the Berry curvature dipole of twisted bilayer $\mathrm{WTe}_{2}$ can be strongly enhanced.

\section{RESULTS \\ Twisted bilayer WTe}

The bilayer WTe $\mathrm{H}_{2}$ exhibits an orthorhombic lattice, the optimized lattice constants are calculated to be $a_{1}=3.447 \AA$ and $a_{2}=$ $6.284 \AA$. We construct a series of twisted bilayer WTe $\mathrm{H}_{2}$ structures based on the method described in Ref. ${ }^{21}$. For simplicity, we start with the normal stacking of perfect bilayer $\mathrm{WTe}_{2}$. As shown in Fig. 1 , to construct the twisted bilayer $\mathrm{WTe}_{2}$, a supercell lattice as bottom layer is built, where $\mathbf{b}_{1}$ and $\mathbf{b}_{2}$ are superlattice basis vectors generated by primitive basis vectors $\mathbf{a}_{1}$ and $\mathbf{a}_{2}$. Correspondingly, the supercell basis vectors of top layer share a mirror plane $\mathcal{M}_{x}$ with bottom layer, which are indicated by $\mathbf{b}_{1}^{\prime}$ and $\mathbf{b}_{2}^{\prime}$ in Fig. 1. Let $\mathbf{b}_{1}=m \mathbf{a}_{1}+n \mathbf{a}_{2}$ and $\mathbf{b}_{2}=p \mathbf{a}_{1}+q \mathbf{a}_{2}$, in which $m, n$, $p$ and $q$ are integers, then the twist angle satisfies $\theta=2 \arctan \left(m\left|\mathbf{a}_{1}\right| / n\left|\mathbf{a}_{2}\right|\right)$. The twisted bilayer structure is formed by rotating the top layer around the original point by angle $\theta$ and translate the top layer by vector $\mathbf{b}_{2}$, while the bottom layer remains fixed. To ensure that the twisted bilayer structure is commensurate, it has to be made sure that $\mathbf{b}_{1}$ is perpendicular to $\mathbf{b}_{2}$. However, this condition is hard to fully meet because of the non-particularity of lattice constants. Approximately, we use a looser condition, for example, the angle between $\mathbf{b}_{1}$ and $\mathbf{b}_{2}$ ranging from $89^{\circ}$ to $91^{\circ}$ is acceptable. It is important to note that the twist angle will change slightly after structural relaxation. More details on the forming process of twisted bilayer for orthorhombic lattice can refer to Ref. ${ }^{21}$.

Table 1 gives the optimized twist angle, formation energy and average interlayer distance for our constructed twisted bilayer $\mathrm{WTe}_{2}$. Despite the twist angles ranging from $12^{\circ}$ to $73^{\circ}$, these systems share pretty close formation energies and average interlayer distances. It is noted that the interlayer spacing in twisted bilayer $\mathrm{WTe}_{2}$ is corrugated, and the average interlayer distance is slightly larger than that in perfect bilayer system

\footnotetext{
${ }^{1}$ Songshan Lake Materials Laboratory, Dongguan, Guangdong, China. ${ }^{2}$ Beijing National Laboratory for Condensed Matter Physics, and Institute of Physics, Chinese Academy of
} Sciences, Beijing, China. ${ }^{3}$ School of Physical Sciences, University of Chinese Academy of Sciences, Beijing, China. ${ }^{凶}$ email: hmweng@iphy.ac.cn 


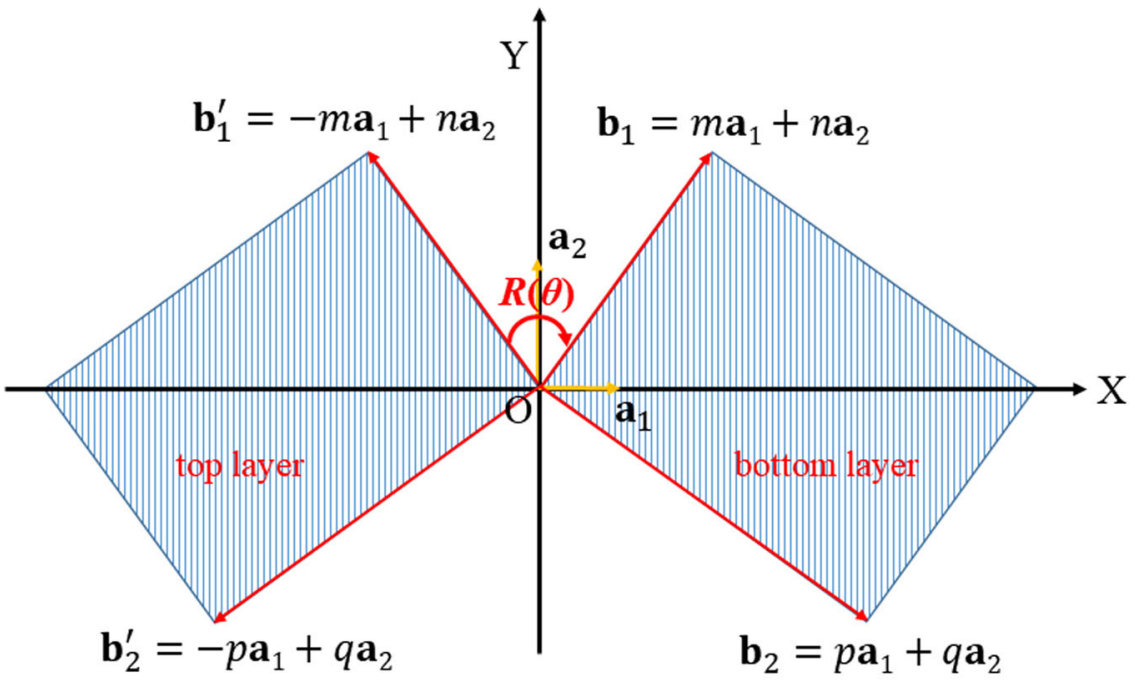

Fig. 1 Forming process of twisted bilayer. Schematics of the forming process of a twisted bilayer for orthorhombic lattice. $\mathbf{a}_{1}$ and $\mathbf{a}_{2}$ are basis vectors for primitive cell, while $\mathbf{b}_{1}$ and $\mathbf{b}_{2}$ are the supercell basis vectors of the bottom layer, $\mathbf{b}_{1}^{\prime}$ and $\mathbf{b}_{2}^{\prime}$ are the supercell basis vectors of the top layer before twisting. $m, n, p$ and $q$ are integers, and it is required that $\mathbf{b}_{1}$ is perpendicular to $\mathbf{b}_{2}$.

Table 1. Parameters of twisted bilayer $\mathrm{WTe}_{2}$.

\begin{tabular}{llllll}
\hline & $(m, n)$ & $(p, q)$ & $\theta$ & $\Delta E\left(\mathrm{meV} / \AA^{2}\right)$ & $\Delta Z(\AA)$ \\
\hline $\mathrm{W}_{344} \mathrm{Te}_{688}$ & $(1,5)$ & $(17,-1)$ & $12.3^{\circ}$ & 6.37 & 3.296 \\
$\mathrm{~W}_{168} \mathrm{Te}_{336}$ & $(2,5)$ & $(8,-1)$ & $25.7^{\circ}$ & 6.62 & 3.275 \\
$\mathrm{~W}_{60} \mathrm{Te}_{120}$ & $(1,2)$ & $(7,-1)$ & $29.4^{\circ}$ & 6.67 & 3.284 \\
$\mathrm{~W}_{68} \mathrm{Te}_{136}$ & $(2,3)$ & $(5,-1)$ & $40.1^{\circ}$ & 6.77 & 3.306 \\
$\mathrm{~W}_{116} \mathrm{Te}_{232}$ & $(5,6)$ & $(4,-1)$ & $49.1^{\circ}$ & 6.55 & 3.307 \\
$\mathrm{~W}_{92} \mathrm{Te}_{184}$ & $(4,3)$ & $(5,-2)$ & $72.3^{\circ}$ & 6.83 & 3.324 \\
\hline
\end{tabular}

The optimized twist angles $\theta$, formation energies $\Delta E$ and average interlayer distances $\Delta Z$ for various twisted bilayer WTe

$(2.888 \AA)$, suggesting a weakening of interlayer coupling. Besides, the relatively smaller formation energy means that the twisted bilayer $\mathrm{WTe}_{2}$ may form in an efficient way.

Compared to the twisted bilayer graphene, the twisted bilayer $\mathrm{WTe}_{2}$ exhibits much more complicated Moiré patterns since one monolayer $\mathrm{WTe}_{2}$ consists of one layer of W atoms and two layers of Te atoms. Figure 2a, b depicts the top and side views of the optimized superlattice for twisted bilayer $\mathrm{WTe}_{2}$ with twist angle $\theta=29.4^{\circ}$ (the structures with other twist angles are given in Supplementary Fig. 1). Such complicated Moiré superlattice leads to an intricate electronic structure in twisted bilayer $\mathrm{WTe}_{2}$. Take the system with twist angle $\theta=29.4^{\circ}$ as an example, there exist extensive band anticrossings and band inversions around the Fermi level, as shown in Fig. 2c. With spin-orbit coupling (SOC) being considered, the band structure becomes more intricate, which can be seen from Fig. $2 \mathrm{~d}$. Similarly, the band structures with other twist angles also exhibit complicated band structures around the Fermi level, as shown in Supplementary Fig. 2. In our calculations, it is gapless for the constructed twisted bilayer $\mathrm{WTe}_{2}$ systems. However, it is found experimentally that there is small band gap in the twisted bilayer $\mathrm{WTe}_{2}$ for some twist angles ${ }^{22}$. This inconsistency may be due to the PBE functional underestimating the band gap in band structure calculation. Besides, the difference in the twist angles between calculation and experiment may be another reason for this contradiction.

\section{Giant nonlinear Hall effect}

The multiple bands cross or nearly cross in momentum space may bring about large gradient of Berry curvature around the band edges and result in strong nonlinear Hall response ${ }^{2}$. Next we focus on the twisted bilayer $\mathrm{WTe}_{2}$ with twist angle $\theta=29.4^{\circ}$, which possesses the smallest number of atoms in a superlattice, to calculate its Berry curvature dipole and estimate the nonlinear Hall effect.

The Berry curvature dipole $D_{x z}$ and $D_{y z}$ of twisted bilayer WTe $\left(\theta=29.4^{\circ}\right)$ as a function of the chemical potential are shown in Fig. 3a. For a two-dimensional system, the Berry curvature dipole takes the unit of length. It is noted that in perfect bilayer WTe ${ }_{2}$ system, due to the presence of $\mathcal{M}_{y}$ mirror plane, only the $y$ component of Berry curvature dipole is nonzero. However, the introduction of twist can break this symmetry, so the $x$ component of Berry curvature dipole is also nonzero in the twisted bilayer $\mathrm{WTe}_{2}$. It is clear that the Berry curvature dipole $D_{x z}$ and $D_{y z}$ exhibit drastic oscillating behavior near the Fermi level, and $D_{x z}$ and $D_{y z}$ can switch their signs dramatically within a very narrow energy region. On the other hand, we find that the magnitude of Berry curvature dipole in our considered twisted bilayer $\mathrm{WTe}_{2}$ is much larger than that in previous reports ${ }^{3,4,11-16}$. For example, the peak of $D_{y z}$ locates near the Fermi level, which is calculated to be $\sim 1400 \AA$. As a comparison, the Berry curvature dipole in monolayer or bilayer WTe $e_{2}$ is estimated to be in the order of $10 \AA^{3,11}$, and $25 \AA$ in strained twisted bilayer $\mathrm{WSe}_{2}{ }^{14}, \sim 200 \AA$ in strained twisted bilayer graphene ${ }^{12}, \sim 700 \AA$ in artificially corrugated bilayer graphene ${ }^{16}$.

To have a clearly understanding of the features of Berry curvature dipole in the twisted bilayer $\mathrm{WTe}_{2}$, we analyze the band structure and the distribution of Berry curvature. In general, the large Berry curvature appears at the band edge, as displayed in Fig. $3 b$, where shows the band structure and Berry curvature $\Omega_{n k, z}$ along the $\Gamma-Y$ line. As can be seen from Fig. $3 b$, the entanglement of multiple bands around the Fermi level causes a complicated distribution of Berry curvature, while the large Berry curvature dipole arises from the drastic change of Berry curvature in momentum space, as indicated by Eq. (2). We find that the peaks of Berry curvature dipole shown in Fig. 3a mainly come from the contribution of the Berry curvature near the band edge shown in Fig. 3b, for instance, the peaks of Berry curvature dipole originate from the band edges at corresponding energy about $-0.02,-0.01,0.0$ and $0.02 \mathrm{eV}$. 
(a)

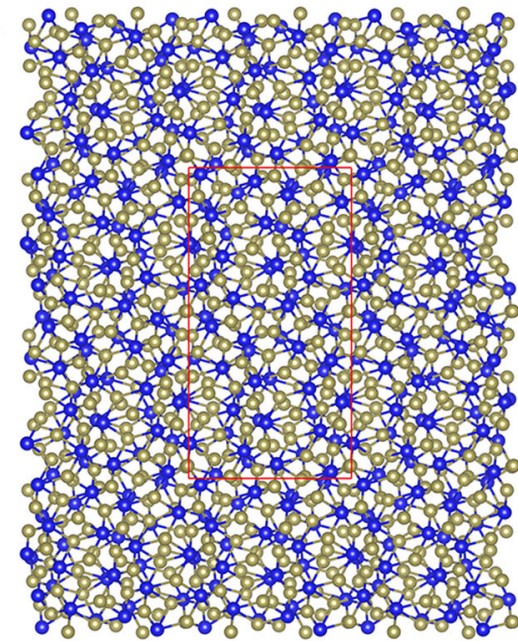

(b)

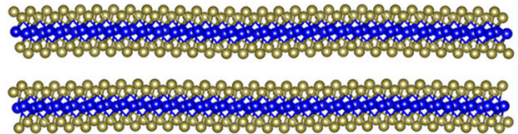

(c)

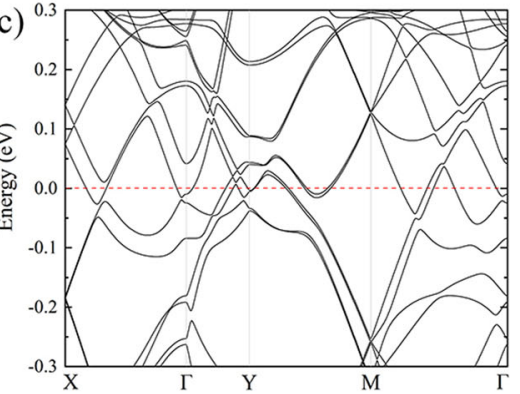

(d)

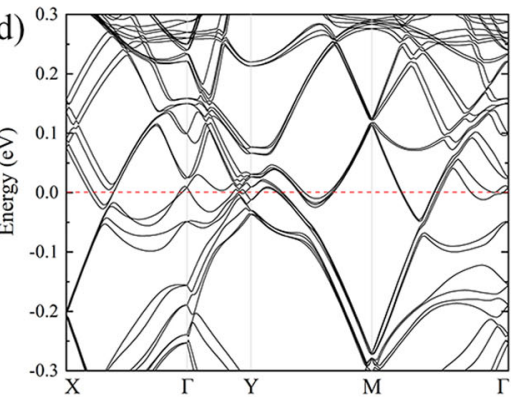

Fig. 2 Atomic structure and band structure of twisted bilayer $\mathbf{W T e} \mathbf{2}_{\mathbf{2}} \mathbf{a}, \mathbf{b}$ The Moiré superlattice structure of twisted bilayer WTe $\left(\theta=29.4^{\circ}\right)$ in top view and side view, respectively. The red rectangle indicates the supercell. The blue and yellow balls represent $W$ and Te atoms, respectively. c, d The band structure of twisted bilayer $\mathrm{WTe}_{2}\left(\theta=29.4^{\circ}\right)$ without and with SOC, respectively.

When the Fermi level is set to the charge neutral point, we plot the Berry curvature $\Omega_{z}$ and the corresponding density of Berry curvature dipole $d_{\mathrm{yz}}$ along the $\mathrm{Y}-\Gamma-\mathrm{Y}$ direction in Fig. $3 c, \mathrm{~d}$, respectively. Here, the density of Berry curvature dipole in momentum space is defined as $d_{b d}=\sum f_{n k} \partial \Omega_{n k, d} / \partial k_{b}$. Clearly, the Berry curvature and $d_{\mathrm{yz}}$ mainly distribute in the region near the $Y$ point, and the tremendous change of Berry curvature contributes large magnitudes to $d_{\mathrm{yz}}$. Furthermore, it is found that the pronounced peaks (marked by " $A$ ") shown in Fig. 3c, $d$ indeed come from the contribution of band edge indicated by the red circle in Fig. 3b. As the Fermi level is shifted from the charge neutral point, the Fermi level go through multiple band anticrossings in a narrow energy region, leading to the dramatic sign change of Berry curvature dipole around the charge neutral point.

As mentioned above, the Berry curvature dipole in twisted bilayer $\mathrm{WTe}_{2}$ exhibits dramatically oscillating behavior near the charge neutral point. This indicates that the strong nonlinear Hall effect in our considered system may be observed at low temperatures. For clarity, we calculate the temperature dependence of Berry curvature dipole $D_{y z}$ for twisted bilayer $\mathrm{WTe}_{2}$ $\left(\theta=29.4^{\circ}\right)$ and perfect bilayer $\mathrm{WTe}_{2}$ according to $D_{y z}(T)=\int D_{y z}(E)(-\partial f / \partial E) d E$. Here, for simplicity, we fix the Fermi level to the charge neutral point. As shown in Fig. 4, the Berry curvature dipole $D_{y z}$ of perfect bilayer $\mathrm{WTe}_{2}$ decreases as the temperature raises. This is qualitatively consistent with the experimental result in few-layer $\mathrm{WTe}_{2}{ }^{4}$, where the nonlinear Hall response decreases monotonically with increasing temperature. When temperature is larger than $20 \mathrm{~K}$, the Berry curvature dipole $D_{y z}$ for the twisted bilayer $\mathrm{WTe}_{2}\left(\theta=29.4^{\circ}\right)$ is slightly larger than that in perfect bilayer $\mathrm{WTe}_{2}$. However, the Berry curvature dipole $D_{y z}$ for the twisted bilayer increases rapidly when temperature is less than $20 \mathrm{~K}$. These results suggest that one could detect a very strong nonlinear Hall response at low temperatures in twisted bilayer $\mathrm{WTe}_{2}$. On the other hand, the Berry curvature dipole in twisted bilayer $\mathrm{WTe}_{2}$ is sensitively dependent on the Fermi level, its magnitude and sign can be switched dramatically within a very narrow energy region. The large magnitude and highly tunable characteristics of Berry curvature dipole in twisted bilayer $\mathrm{WTe}_{2}$ provide an excellent platform to investigate the nonlinear Hall effect.

\section{DISCUSSION}

In this work, we focus on the nonlinear Hall effect in twisted bilayer $\mathrm{WTe}_{2}$ with twist angle $\theta=29.4^{\circ}$. It is worth understanding the angle dependence of the nonlinear Hall effect in twisted bilayer $\mathrm{WTe}_{2}$ systems. For comparison, we calculate the energy dependence of Berry curvature dipole for twisted bilayer $\mathrm{WTe}_{2}$ with twist angle $\theta=40.1^{\circ}$, the results are shown in Supplementary Fig. 3a. Similar to the system with twist angle $\theta=29.4^{\circ}$, the Berry curvature dipole in twisted bilayer $\mathrm{WTe}_{2}\left(\theta=40.1^{\circ}\right)$ also exhibits large magnitude (larger than $1500 \AA$ ) and drastic oscillating behavior. However, considering the large computational costs, the nonlinear Hall effect of other twist angle systems is not calculated. Nevertheless, we can make a simple prediction for the features of the nonlinear Hall effect in these systems according to the band structure. For example, there also exist rich band crossings in the case of twist angles $\theta=12.3^{\circ}$ and $\theta=25.7^{\circ}$ (see Supplementary Fig. 2). The complicated band structure in a narrow energy region brings about the dramatically oscillating behavior of nonlinear Hall response in these twisted bilayer WTe systems. As a comparison, the band structure in the perfect bilayer $\mathrm{WTe}_{2}$ is much simpler (see Supplementary Fig. 2a), and the energy dependence of Berry curvature dipole for perfect bilayer $\mathrm{WTe}_{2}$ is also much smoother (see Supplementary Fig. 3b). Moreover, the entanglement of multiple bands around the Fermi level indicates the drastic change of Berry curvature in momentum space, which may lead to the giant Berry curvature dipole in these twisted bilayer $\mathrm{WTe}_{2}$ systems. Of course, the predictions mentioned above may be not applicable to the small twist angle systems. On the other hand, the contributions to the nonlinear Hall effect can be divided as intrinsic (geometric) and extrinsic (disorder-induced) contributions ${ }^{23}$. Here, we focus on the intrinsic part of the nonlinear Hall conductivity, which is related to the Berry curvature dipole. However, it is revealed that disorder-induced extrinsic part has more important contribution to the nonlinear Hall effect in 

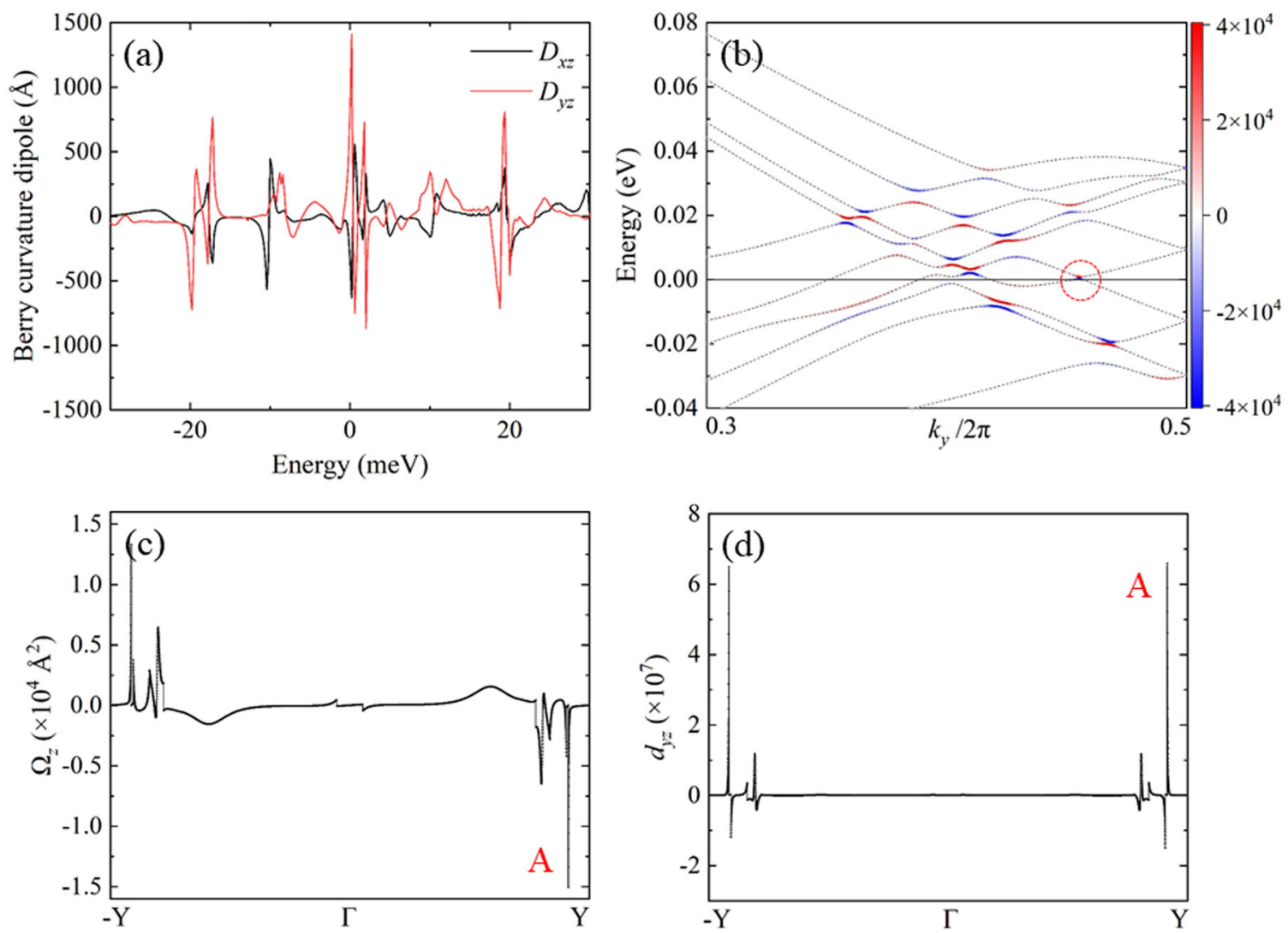

Fig. 3 Nonlinear Hall effect in twisted bilayer $\mathbf{W T e}_{2}$. a The Fermi energy dependence of Berry curvature dipole for twisted bilayer WTe 2 $\left(\theta=29.4^{\circ}\right)$. b Band structure and Berry curvature $\Omega_{n k, z}\left(\AA^{2}\right)$ along $\Gamma-Y$ line. The calculated Fermi level is set to be zero. c, d Berry curvature $\Omega_{z}$ and Berry curvature dipole density $d_{\mathrm{yz}}$ along $\mathrm{Y}-\Gamma-\mathrm{Y}$ line, respectively.

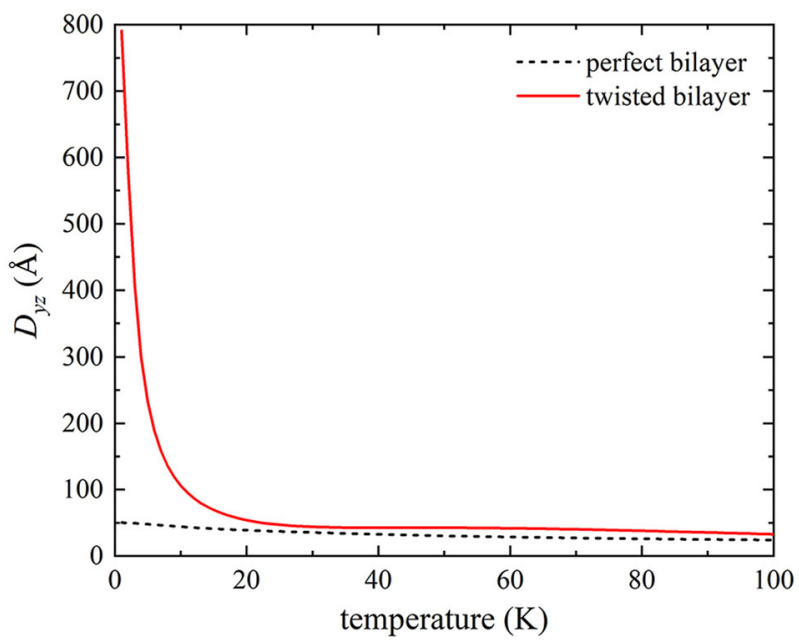

Fig. 4 Temperature dependence of Berry curvature dipole. The temperature dependence of Berry curvature dipole $D_{y z}$ for twisted bilayer $\mathrm{WTe}_{2}\left(\theta=29.4^{\circ}\right)$ and prefect bilayer $\mathrm{WTe}_{2}$.

some recent works ${ }^{24-26}$. In twisted bilayer systems, the nonuniformity of twist angle across the sample is believed to be the main source of disorder ${ }^{27}$, which may have pronounced contribution to the extrinsic part of the nonlinear Hall effect. These issues deserve further study.

In summary, we have predicted that a giant nonlinear Hall effect can exist in twisted bilayer $\mathrm{WTe}_{2}$ system. We show that the twist can greatly change the band structure of bilayer $\mathrm{WTe}_{2}$. There exist abundant band anticrossings and band inversions around the Fermi level in the twisted bilayer $\mathrm{WTe}_{2}$, which brings a strong nonlinear Hall signal in this system. The Berry curvature dipole of twisted bilayer $\mathrm{WTe}_{2}\left(\theta=29.4^{\circ}\right)$ can reach up to $\sim 1400 \AA$, much larger than that in previously reported nonlinear Hall systems. In addition, the nonlinear Hall effect in twisted bilayer $\mathrm{WTe}_{2}$ exhibits dramatically oscillating behavior due to the complicated distribution of Berry curvature around the Fermi level. Our results show that the twisted bilayer $\mathrm{WTe}_{2}$ can become an excellent platform to investigate the nonlinear Hall effect.

\section{METHODS}

\section{Nonlinear Hall effect}

The nonlinear Hall effect originates from the dipole moment of Berry curvature over the occupied states. In a system with time-reversal symmetry but broken inversion symmetry, when an oscillating electric field $E_{c}=\operatorname{Re}\left\{\xi_{c} e^{i \omega t}\right\}$ is applied, a transverse response current $j_{a}=$ $\operatorname{Re}\left\{j_{a}^{(0)}+j_{a}^{(2 \omega)} e^{2 i \omega t}\right\}$ can be generated, where $j_{a}^{(0)}=x_{a b c} \xi_{b} \xi_{c}^{*}$ and $j_{a}^{(2 \omega)}=$ $X_{a b c} \xi_{b} \xi_{c}$ are rectified current and second-harmonic current, respectively. The nonlinear conductivity tensor $X_{a b c}$ is associated with the Berry curvature dipole ${ }^{2}$ as follows:

$X_{a b c}=-\varepsilon_{a d c} \frac{e^{3} \tau}{2 \hbar^{2}(1+i \omega \tau)} D_{b d}$.

Here, $D_{b d}$ is the Berry curvature dipole, $\varepsilon_{a d c}$ is the Levi-Civita symbol and $\tau$ is the relaxation time. The Berry curvature dipole can be written $a^{2}$

$D_{b d}=\sum_{n} \int_{k} f_{n k} \frac{\partial \Omega_{n k, d}}{\partial k_{b}}$, 
where $f_{n k}$ is the Fermi-Dirac distribution function, and $\Omega_{n k, d}$ is the $d$ component of the Berry curvature. For a two-dimensional system, only the out-of-plane component $(z)$ of Berry curvature is nonzero, which is given by ${ }^{28}$

$\Omega_{n k, z}=2 i \sum_{m \neq n} \frac{\left\langle n\left|\partial H / \partial k_{x}\right| m\right\rangle\left\langle m\left|\partial H / \partial k_{y}\right| n\right\rangle}{\left(E_{n}-E_{m}\right)^{2}}$,

where $E_{n}$ and $|n\rangle$ are eigenvalues and eigenwave functions, respectively.

\section{First-principles calculations}

The first-principles calculations are performed by using the Vienna ab initio simulation package (VASP) ${ }^{29}$ with the projector-augmented wave potential method ${ }^{30-32}$. The exchange-correlation potential is described using the generalized gradient approximation (GGA) in the Perdew-Burke-Ernzerhof (PBE) form ${ }^{33}$. Spin-orbit coupling is taken into account self-consistently. The energy cutoff of the plane-wave basis set is $350 \mathrm{eV}$. A vacuum region larger than $15 \AA$ is applied to ensure no interaction between the slab and its image. In our optimization, all structures are fully relaxed until the force on each atom is less than $0.02 \mathrm{eV} / \AA \AA$. The Van der Waals interactions between the adjacent layers are taken into account by using zero damping DFT-D3 method of Grimme ${ }^{34}$. The maximally localized Wannier function $s^{35-37}$ for the $d$ orbitals of W and $p$ orbitals of Te are generated to compute the Berry curvature and Berry curvature dipole. For the integral of Berry curvature dipole, the first Brillouin zone is sampled by very dense $\mathrm{k}$ grids to get a convergent result. Considering the large computational cost, we separate the first Brillouin zone into multiple blocks, and the convergence test is carried out independently for each block. For example, some blocks are sampled with the k-point separation of $5 \times 10^{-5} \AA^{-1}$.

\section{DATA AVAILABILITY}

The data that support the findings of this study are available from the corresponding authors upon reasonable request.

\section{CODE AVAILABILITY}

The codes that were used here are available upon request to the corresponding author.

Received: 18 May 2021; Accepted: 28 November 2021; Published online: 14 December 2021

\section{REFERENCES}

1. Nagaosa, N., Sinova, J., Onoda, S., MacDonald, A. H. \& Ong, N. P. Anomalous Hall effect. Rev. Mod. Phys. 82, 1539-1592 (2010).

2. Sodemann, I. \& Fu, L. Quantum nonlinear Hall effect induced by Berry curvature dipole in time-reversal invariant materials. Phys. Rev. Lett. 115, 216806 (2015).

3. $\mathrm{Ma}, \mathrm{Q}$. et al. Observation of the nonlinear Hall effect under time-reversalsymmetric conditions. Nature 565, 337-342 (2019).

4. Kang, K., Li, T., Sohn, E., Shan, J. \& Mak, K. F. Nonlinear anomalous Hall effect in few-layer $\mathrm{WTe}_{2}$. Nat. Mater. 18, 324-328 (2019).

5. Zhang, Y., Sun, Y. \& Yan, B. Berry curvature dipole in Weyl semimetal materials: an ab initio study. Phys. Rev. B 97, 041101(R) (2018).

6. Singh, S., Kim, J., Rabe, K. M. \& Vanderbilt, D. Engineering Weyl phases and nonlinear Hall effects in $\mathrm{T}_{d}-\mathrm{MoTe}_{2}$. Phys. Rev. Lett. 125, 046402 (2020).

7. Tiwari, A. et al. Giant c-axis nonlinear anomalous Hall effect in $T_{d}-M_{0} e_{2}$ and WTe 2 . Nat. Commun. 12, 2049 (2021).

8. Kumar, D. et al. Room-temperature nonlinear Hall effect and wireless radiofrequency rectification in Weyl semimetal TalrTe 4 . Nat. Nanotech. 16, 421-425 (2021).

9. Du, Z. Z., Wang, C. M., Lu, H. Z. \& Xie, X. C. Band signatures for strong nonlinear Hall effect in bilayer WTe 2 . Phys. Rev. Lett. 121, 266601 (2018).

10. Facio, J. I. et al. Strongly enhanced Berry dipole at topological phase transitions in BiTel. Phys. Rev. Lett. 121, 246403 (2018).

11. Zhang, Y., van den Brink, J., Felser, C. \& Yan, B. Electrically tuneable nonlinear anomalous Hall effect in two-dimensional transition-metal dichalcogenides $\mathrm{WTe}_{2}$ and $\mathrm{MoTe}_{2}$. 2D Matter 5, 044001 (2018).

12. Zhang, C. P. et al. Giant nonlinear Hall effect in strained twisted bilayer graphene. Preprint at https://arxiv.org/abs/2010.08333v1 (2020).
13. Hu, J. X., Zhang, C. P., Xie, Y. M. \& Law, K. T. Nonlinear Hall effects in strained twisted bilayer WSe $\mathrm{W}_{2}$. Preprint at https://arxiv.org/abs/2004.14140 (2020).

14. Huang, M. et al. Giant nonlinear Hall effect in twisted WSe $\mathrm{W}_{2}$. Preprint at https:// arxiv.org/abs/2006.05615 (2020).

15. Qin, M. S. et al. Strain tunable Berry curvature dipole, orbital magnetization and nonlinear Hall effect in WSe 2 monolayer. Chin. Phys. Lett. 38, 017301 (2021).

16. Ho, S. C. et al. Hall effects in artificially corrugated bilayer graphene without breaking time-reversal symmetry. Nat. Electron. 4, 116-125 (2021).

17. Battilomo, R., Scopigno, N. \& Ortix, C. Berry curvature dipole in strained graphene: a Fermi surface warping effect. Phys. Rev. Lett. 123, 196403 (2019).

18. Cao, Y. et al. Unconventional superconductivity in magic-angle graphene superlattices. Nature 556, 43-50 (2018).

19. Cao, Y. et al. Correlated insulator behaviour at half-filling in magic-angle graphene superlattices. Nature 556, 80-84 (2018).

20. Wang, L. et al. Correlated electronic phases in twisted bilayer transition metal dichalcogenides. Nat. Mater. 19, 861-866 (2020).

21. Pan, D., Wang, T. C., Xiao, W., Hu, D. \& Yao, Y. Simulations of twisted bilayer orthorhombic black phosphorus. Phys. Rev. B 96, 041411(R) (2017).

22. Lüpke, F. et al. Quantum spin Hall edge states in twisted-bilayer $1 \mathrm{~T}^{\prime}-\mathrm{WTe} \mathrm{e}_{2}$. Preprint at https://arxiv.org/abs/2010.13699v1 (2020).

23. Du, Z. Z., Lu, H. Z. \& Xie, X. C. Nonlinear Hall effects. Nat. Rev. Phys. 3, 744-752 (2021).

24. Du, Z. Z., Wang, C. M., Li, S., Lu, H. Z. \& Xie, X. C. Disorder-induced nonlinear Hall effect with time-reversal symmetry. Nat. Commun. 10, 3047 (2019).

25. Xiao, C., Du, Z. Z. \& Niu, Q. Theory of nonlinear Hall effects: Modified semiclassics from quantum kinetic. Phys. Rev. B 100, 165422 (2019).

26. Du, Z. Z., Wang, C. M., Sun, H. P., Lu, H. Z. \& Xie, X. C. Quantum theory of the nonlinear Hall effect. Nat. Commun. 12, 5038 (2021).

27. Wilson, J. H., Fu, Y. X., Sarma, S. D. \& Pixley, J. H. Disorder in twisted bilayer graphene. Phys. Rev. Res. 2, 023325 (2020).

28. Xiao, D., Chang, M. C. \& Niu, Q. Berry phase effects on electronic properties. Rev. Mod. Phys. 82, 1959-2007 (2010).

29. Kresse, G. \& Hafner, J. Ab initio molecular dynamics for liquid metals. Phys. Rev. B 47, 558(R) (1993)

30. Blöchl, P. E. Projector augmented-wave method. Phys. Rev. B 50, 17953 (1994).

31. Kresse, G. \& Furthmüller, J. Efficient iterative schemes for ab initio total-energy calculations using a plane-wave basis set. Phys. Rev. B 54, 11169 (1996)

32. Kresse, G. \& Joubert, D. From ultrasoft pseudopotentials to the projector augmented-wave method. Phys. Rev. B 59, 1758 (1999).

33. Perdew, J. P., Burke, K. \& Ernzerhof, M. Generalized gradient approximation made simple. Phys. Rev. Lett. 77, 3865 (1996).

34. Grimme, S., Antony, J., Ehrlich, S. \& Krieg, S. A consistent and accurate ab initio parametrization of density functional dispersion correction (dft-d) for the 94 elements H-Pu. J. Chem. Phys. 132, 154104 (2010).

35. Marzari, N. \& Vanderbilt, D. Maximally localized generalized Wannier functions for composite energy bands. Phys. Rev. B 56, 12847 (1997).

36. Souza, I., Marzari, N. \& Vanderbilt, D. Maximally localized Wannier functions for entangled energy bands. Phys. Rev. B 65, 035109 (2001).

37. Mostofi, A. A. et al. An updated version of wannier90: a tool for obtaining maximally-localised Wannier functions. Comput. Phys. Commun. 185, 2309-2310 (2014)

\section{ACKNOWLEDGEMENTS}

This work was supported by the National Natural Science Foundation of China (Grant Nos. 11925408 and 12005153), the National Key Research and Development Program of China (Grant Nos. 2016YFA0300600 and 2018YFA0305700), the K. C. Wong Education Foundation (GJTD-2018-01), the Beijing Natural Science Foundation (Z180008), the Beijing Municipal Science and Technology Commission (No Z191100007219013) and the Strategic Priority Research Program of Chinese Academy of Sciences (Grant No. XDB33000000). The computational resource is provided by the Platform for Data-Driven Computational Materials Discovery in Songshan Lake material Laboratory. The authors acknowledge the discussion with Prof. K. T. Law and Prof. N. Wang.

\section{AUTHOR CONTRIBUTIONS}

Z.H.H. performed the calculation. Both Z.H.H. and H.M.W. proposed the basic idea and wrote the paper.

\section{COMPETING INTERESTS}

The authors declare no competing interests. 


\section{ADDITIONAL INFORMATION}

Supplementary information The online version contains supplementary material available at https://doi.org/10.1038/s41535-021-00403-9.

Correspondence and requests for materials should be addressed to Hongming Weng.

Reprints and permission information is available at http://www.nature.com/ reprints

Publisher's note Springer Nature remains neutral with regard to jurisdictional claims in published maps and institutional affiliations.
Open Access This article is licensed under a Creative Commons Attribution 4.0 International License, which permits use, sharing, adaptation, distribution and reproduction in any medium or format, as long as you give appropriate credit to the original author(s) and the source, provide a link to the Creative Commons license, and indicate if changes were made. The images or other third party material in this article are included in the article's Creative Commons license, unless indicated otherwise in a credit line to the material. If material is not included in the article's Creative Commons license and your intended use is not permitted by statutory regulation or exceeds the permitted use, you will need to obtain permission directly from the copyright holder. To view a copy of this license, visit http://creativecommons. org/licenses/by/4.0/.

(c) The Author(s) 2021 